\title{
A Phase I/III, multicenter, open-label trial of talimogene laherparepvec (T-VEC) in combination with pembrolizumab for the treatment of unresected, stage IIIb-IV melanoma (MASTERKEY-265)
}

\author{
Georgina V Long ${ }^{1 *}$, Reinhard Dummer ${ }^{2}$, Antoni Ribas ${ }^{3}$, Igor Puzanov ${ }^{4}$, Olivier Michielin ${ }^{5}$, Ari VanderWalde , \\ Robert HI Andtbacka ${ }^{7}$, Jonathan Cebon ${ }^{8}$, Eugenio Fernandez ${ }^{9}$, Josep Malvehy ${ }^{10}$, Anthony J Olszanski ${ }^{11}$, \\ Thomas F Gajewski ${ }^{12}$, John M Kirkwood ${ }^{13}$, Olga Kuznetsova ${ }^{14}$, Lisa Chen ${ }^{15}$, David R Kaufman ${ }^{14}$, Jeffrey Chou ${ }^{15}$, \\ F Stephen $\mathrm{Hodi}^{16}$
}

From 30th Annual Meeting and Associated Programs of the Society for Immunotherapy of Cancer (SITC 2015) National Harbor, MD, USA. 4-8 November 2015

\section{Background}

T-VEC is a herpes simplex virus-1-based oncolytic immunotherapy designed to selectively replicate in tumors, produce GM-CSF, and stimulate antitumor immune responses. OPTiM, a Phase III trial of T-VEC vs GM-CSF in unresectable stage IIIB-IV melanoma, improved the primary endpoint of durable response rate (DRR) in the T-VEC arm (16 vs 2\%).[1] Pembrolizumab, a human programmed death receptor-1 (PD-1)-blocking antibody approved for the treatment of advanced metastatic or unresectable melanoma, has demonstrated superiority over the CTLA-4-blocking antibody ipilimumab in patients with stage III or IV melanoma that received no more than one prior line of systemic therapy (PFS HR 0.58, OS HR 0.63-0.69).[2] Combining T-VEC with pembrolizumab may enhance antitumor immune responses vs either therapy alone. Here, we describe a Phase Ib/III study assessing the safety and efficacy of T-VEC + pembrolizumab in unresected stage IIIB-IV melanoma. Twenty-one patients enrolled in Phase Ib December 2014 through March 2015 at 11 institutions in Australia, Spain, Switzerland, and the United States.

'Melanoma Institute Australia and The University of Sydney, Sydney, Australia Full list of author information is available at the end of the article

\section{Methods}

Primary objective for Phase Ib: assess dose-limiting toxicities of T-VEC + pembrolizumab. Key secondary objectives for Phase Ib: best OR, DRR, duration of response, disease control rate, PFS by investigator using modified immune-related response criteria (irRC), OS, treatmentemergent/related AEs, and potential blood/tumor biomarkers for response/resistance to combination treatment. Key eligibility criteria for Phase Ib: stage IIIB-IV melanoma naïve to systemic treatment (except adjuvant), injectable lesions, ECOG PS $0-1$, no active cerebral metastases, no autoimmunity/immunosuppression, and no active herpetic infection. In Phase Ib, T-VEC is injected into cutaneous, subcutaneous, or nodal lesions at up to $4 \mathrm{~mL}$ of $10^{6}$ plaque forming units (PFU)/mL day 1 , then at up to $4 \mathrm{~mL}$ of $10^{8} \mathrm{PFU} / \mathrm{mL}$ day 22 and Q2W. Pembrolizumab is given $200 \mathrm{mg}$ IV Q2W. Treatment with both therapies continues until (whichever comes first): CR or PD per irRC, intolerance, for up to 2 yrs or, for T-VEC, when there are no longer injectable lesions. The randomized portion of the study comparing T-VEC + pembrolizumab to pembrolizumab alone was originally designed as a Phase II study. An updated Phase III design will be presented.

\section{Trial registration}

ClinicalTrials.gov identifier NCT02263508. 


\section{Authors' details}

'Melanoma Institute Australia and The University of Sydney, Sydney,

Australia. ${ }^{2}$ University Hospital of Zurich, Zurich, Switzerland. ${ }^{3}$ University of California at Los Angeles Medical Center, Los Angeles, CA, USA. "Vanderbilt University Medical Center, Nashville, TN, USA. ${ }^{5}$ Centre Hospitalier

Universitaire Vaudois, Lausanne, Switzerland. ${ }^{6}$ The West Clinic, Memphis, TN, USA. ${ }^{7}$ University of Utah Huntsman Cancer Institute, Salt Lake City, UT, USA.

${ }^{8}$ Austin Health, Austin Hospital, Heidelberg, Australia. ${ }^{9}$ Hôpitaux Universitaires de Genève, Geneva, Switzerland. ${ }^{10}$ Hospital Clinic i Provincial de Barcelona, Barcelona, Spain. ${ }^{11}$ Fox Chase Cancer Center, Philadelphia, PA, USA. ${ }^{12}$ The University of Chicago Medicine, Chicago, IL, USA. ${ }^{13}$ University of Pittsburgh Cancer Institute, Pittsburgh, PA, USA. ${ }^{14}$ Merck \& Co., Inc., Kenilworth, NJ, USA. ${ }^{15}$ Amgen Inc., Thousand Oaks, CA, USA. ${ }^{16}$ Dana-Farber Cancer Institute, Boston, MA, USA.

Published: 4 November 2015

\section{References}

1. Andtbacka RH, Kaufman HL, Collichio F, et al: Talimogene Laherparepvec Improves Durable Response Rate in Patients with Advanced Melanoma. J Clin Oncol 2015, doi:10.1200/JCO.2014.58.3377 [epub ahead of print].

2. Robert C, Schachter J, Long GV, et al: Pembrolizumab versus Ipilimumab in Advanced Melanoma. N Engl J Med 2015, 372:2521-2532.

doi:10.1186/2051-1426-3-S2-P181

Cite this article as: Long et al:: A Phase I/III, multicenter, open-label trial of talimogene laherparepvec (T-VEC) in combination with pembrolizumab for the treatment of unresected, stage IIIb-IV melanoma (MASTERKEY-265). Journal for ImmunoTherapy of Cancer 2015 3(Suppl 2):P181.

\section{Submit your next manuscript to BioMed Central} and take full advantage of:

- Convenient online submission

- Thorough peer review

- No space constraints or color figure charges

- Immediate publication on acceptance

- Inclusion in PubMed, CAS, Scopus and Google Scholar

- Research which is freely available for redistribution

Submit your manuscript at www.biomedcentral.com/submit
Ciomed Central 